\title{
Cine de animación made in SPAIN: DOBLAJE Y SUBTITULACIÓN DE ELEMENTOS CULTURALES
}

\author{
Animated Films made in Spain: Dubing and Subtitling of Cultural Elements \\ Films D'ANIMATION MADE IN SPAIN : LA TRADUCTION DES ÉLÉMENTS CULTURELS AU DOUBLAGE \\ ET SOUS-TITRAGE
}

\section{Adrián Fuentes-Luque}

Doctor en Traducción e Interpretación, Universidad de Granada, España.

Profesor titular, Departamento de Filología y Traducción, Universidad Pablo de Olavide, Sevilla, España. afuentes@upo.es http://orcid. org/0000-0001-9717-2519

\section{Rebeca López González} Doctora en Traducción e Interpretación, Universidade de Vigo. Profesora ayudante doctora, Departamento de Traducción y Lingüística, y miembro del grupo de investigación GALMA Observatory for Media Accessibility, Universidade de Vigo, España. rebecalopez@uvigo.es http://orcid. org/0000-0002-9358-7562

\section{RESUMEN}

Si bien los estudios de Traducción se han ocupado de la traducción del cine de animación de acuerdo con los distintos modos de traducción audiovisual (doblaje, subtitulación, etc.), dichos estudios se han centrado casi exclusivamente en las versiones traducidas desde el inglés. Hasta el momento, se ha estudiado poco la traducción al inglés de los elementos culturales contenidos en las películas de animación producidas en español. Este trabajo analiza el doblaje y el subtitulado de tres películas de animación producidas en España: Planet 51 (2009), Las aventuras de Tadeo Jones (2012) y Atrapa la bandera (2015), con el objetivo de conocer qué elementos culturales se han incluido en la versión original en español de cada una de estas producciones y cómo se han traducido en las versiones doblada y subtitulada al inglés. Para ello se desarrolló una metodología de trabajo que partió de la clasificación de los elementos culturales y de diversas estrategias de traducción utilizadas para el doblaje y subtitulado de contenido cultural. Se halló que la mitad de los elementos culturales recopilados exigen la intervención del traductor, y se clasifican en la categoría "medio" y en el subtipo "cinematográfico". El cine es el elemento cultural de peso en el corpus.

Palabras clave: cine de animación; cine en español; traducción audiovisual; doblaje; subtitulación; elementos culturales.

\section{Abstract}

Although Translation Studies have dealt with the translation of animated films into the various modes of audiovisual translation (dubbing, subtitling, etc.), such studies have focused almost exclusively on films translated from English into other languages. So far, there are hardly any studies analysing the translation into English of cultural elements contained in animation films produced in Spanish language. This article analyses the dubbing and subtitling of three animation films produced in Spain -Planet 51 (2009), Las aventuras de Tadeo Jones (2012), and Atrapa la bandera (2015), with the aim of finding out which cultural elements have been included

Recibido: 2019-05-02 / Aceptado: 2019-11-15 / Publicado: 2020-05-05

https://doi.org/10.17533/udea.ikala.v25n02a08 
in the original Spanish version of each of these productions and how they have been translated into the English dubbed and subtitled versions. To this end, a working methodology was developed based on the classification of cultural elements and on the various translation strategies used for dubbing and subtitling cultural content. Results show that almost half of the cultural elements compiled demand the services of a translator. These cases have been classified under the 'Environment' category and the 'Cinematography' subtype. This final subtype has a major impact on the corpus.

Keywords: animation films; films in Spanish; audiovisual translation; dubbing; subtitling; cultural elements.

\section{RÉSUMÉ}

Bien que les études de traduction se soient occupées de la traduction de films d'animation dans les différents modes de traduction audiovisuelle (doublage, sous-titrage, etc.), ces études se sont concentrées presque exclusivement sur les versions traduites de l'anglais. Jusqu'à présent, il n'existe guère d'études analysant la traduction en anglais des éléments culturels contenus dans les films d'animation produits en espagnol. Cet article analyse le doublage et le sous-titrage de trois films d'animation produits en Espagne - Planet 51 (2009), Las aventuras de Tadeo Jones (2012) et Atrapa la bandera (2015) visant à savoir quels éléments culturels ont été inclus dans la version originale espagnole de chacune de ces productions et comment ils ont été traduits dans les versions doublés et sous-titrées en anglais. À cette fin, nous avons élaboré une méthodologie de travail à partir de la classification des éléments culturels et des différentes stratégies de traduction utilisées pour le doublage et le sous-titrage des contenus culturels. Les résultats montrent que presque la moitié des éléments culturels identifiés exigent les services du traducteur sont classés dans la categorie 'Environment', sous-type 'Cinématographique'. Le cinema donc réprésente un élement culturel de poids dans ce corpus.

Mots clés : animation ; cinéma en espagnol ; traduction audiovisuelle ; doublage ; sous-titrage. 


\section{Introducción: los elementos culturales y su clasificación}

El estudio de la repercusión de los elementos culturales en el proceso traslativo ha formado parte de los estudios de traducción desde sus inicios. Las definiciones de "cultura" y de "elemento cultural", y su clasificación en múltiples categorías, con el propósito de orientar a los traductores - profesionales y en formación - en el reconocimiento y la transferencia interlingüística de estos contenidos, han sido el objeto de múltiples trabajos (Baker, 1992; Bassnett, 1991; Hermans, 1999; Katan, 1999; Leppihalme, 1994 y 1997; Mayoral Asensio, 1994; Newmark, 1988; Snell-Hornby, 1988; Vlakhov y Florin, 1970 — citado en Cómitre y Valverde, 2014, p. 72-).

En concreto, el trabajo de Nedergaard-Larsen (1993) para iniciar nuestra reflexión teórica resulta útil por dos motivos: el primero, porque permite establecer un punto de partida del análisis del contenido cultural incluido en los textos audiovisuales, al proponer una distinción entre los elementos culturales insertos en el lenguaje, es decir, intralingüísticos, y los elementos culturales que no se encuentran dentro del lenguaje, o extralingüísticos. Nuestro trabajo se enmarca dentro de esta segunda categoría, con el objetivo de realizar un análisis que va más allá de la lengua. El segundo motivo radica en que Nedergaard-Larsen (1993, p. 207) se centra en el subtitulado del contenido cultural y define una película como un producto creado y enmarcado en una cultura, la cual originará una serie de dificultades de comprensión si se proyecta en otra cultura distinta. Pedersen (2011, p. 43) retoma la etiqueta "extralingüístico" y propone el término "referencia cultural extralingüística”, una nomenclatura que adoptamos también en esta investigación. Dentro de las referencias culturales extralingüísticas hallamos las referencias a la cultura popular, las cuales forman parte del corpus aquí estudiado.

Por lo que respecta a la clasificación de los elementos culturales atendiendo a sus propiedades semánticas, puede citarse la propuesta de Nedergaard-Larsen
(1993, p. 211), que distingue cuatro categorías generales, con sus correspondientes subcategorías: geografía (geografía, meteorología, biología y geografía cultural), historia (edificios, sucesos, personas), sociedad (nivel industrial, organización social, política, condición social y tradiciones) y cultura (religión, educación, medios y actividades culturales de ocio); y la aportación de Pedersen (2011), que incluye doce campos: 1) pesos y medidas, 2) nombres propios, 3) designaciones profesionales, 4) comida y bebida, 5) literatura, 6) gobierno, 7) entretenimiento, 8) educación, 9) deportes, 10) moneda, 11) material técnico, y 12) otros.

Cómitre Narváez (2015, p. 2) recoge, a su vez, dos clasificaciones: la de Ramière (2004), extralingüística, histórica y sociocultural, y la de Vandeweghe (2005), geográfica, etnográfica y sociopolítica, y menciona cómo se tiende a aplicar la propuesta más general de Venuti (1995), que distribuye el contenido cultural a lo largo de un espectro que se desplaza del extremo extranjerizante al domesticador.

Tanto Cómitre Narváez (2015) como Ramière (2004) sostienen que estas clasificaciones del referente cultural se realizaron para un contexto en el que la traducción se desarrolla entre textos impresos, lo cual desatiende las restricciones impuestas por el texto audiovisual.

A pesar de la naturaleza específica de cada uno de estos textos, opinamos que, dentro de las múltiples categorías descritas hasta el momento, la tipología clásica propuesta por Katan (1999, pp. 63-99) permite el análisis de los elementos culturales más allá del condicionante impreso/audiovisual, como se comprueba en el apartado práctico de esta investigación. De hecho, la aplicación de este modelo no impide que se tengan muy presentes ciertos aspectos propios de los textos audiovisuales como, por ejemplo, los canales involucrados en la transmisión del contenido cultural.

Este modelo establece los siguientes epígrafes: 1) el medio (el clima, el hogar, la comida); 2) el comportamiento (acciones y modos de comportarse 
en cada cultura; 3) capacidades, estrategias y destrezas empleadas en la comunicación (comunicación no verbal, rituales, etc.); 4) valores de la sociedad y sus jerarquías; 5) creencias, y 6 ) identidad.

Con el objetivo de describir en profundidad los datos recopilados, se aplica este último modelo y se expande el contenido relativo a la categoría "medio" (clima, hogar, comida), en tanto se halla un número considerable de casos que pueden clasificarse dentro de ella, pero que no pueden delimitarse dentro de las tres etiquetas descritas por Katan (ámbito doméstico, clima y comida). En últimas, el modelo de Katan permite clasificar y conocer la naturaleza de cada uno de los elementos culturales hallados en el corpus.

De esta manera, teniendo presentes los datos encontrados, se amplía la categoría "medio" para incluir las siguientes subcategorías: artístico, cinematográfico, histórico, institucional, literario, lugar, moneda, musical, publicitario y televisivo. Así, se ha podido cuantificar qué elementos se insertan en el ámbito doméstico, el clima y la comida.

Por otra parte, el modelo de Pedersen se aplica aquí para valorar las opciones estratégicas del traductor en cuanto a la modalidad de subtitulación, pero no explica el análisis realizado, en lo que se refiere al doblaje de los elementos culturales comentados más abajo.

Tras describir acá el modelo aplicado en este estudio, el apartado siguiente se ocupa del trasvase del contenido cultural en el proceso de doblaje y subtitulado.

Sin embargo, antes queremos indicar que la literatura centrada en el estudio del doblaje y del subtitulado del cine de animación suele estudiar cómo se efectúa el trasvase del inglés al español. Con esta afirmación, no nos referimos a las capacidades lingüísticas del traductor, o a si el inglés o el español son sus lenguas maternas o no, sino a que muchos de los estudios llevados a cabo sobre ambas modalidades de traducción audiovisual (TAV) suelen desarrollar su análisis en la dirección del inglés hacia el español. Esto se debe a que la producción de animación se ha centrado, en el último siglo, principalmente, en Estados Unidos y el Reino Unido por motivos históricos. Piénsese en la factoría Disney y cómo muchos de sus creadores, como, por ejemplo, Tim Burton, iniciaron sus primeros pasos en la omnipresente multinacional. Esta es una de las razones por las que nuestra atención se desplaza hacia la producción realizada en español y en España. En este sentido, creemos que es conveniente analizar la industria desde otra perspectiva que, a pesar de continuar siendo la centrada en Occidente, ofrezca una visión de qué se produce en español y en España, y cómo se traduce para la distribución internacional en inglés.

\section{La cultura y su traducción audiovisual: doblaje y subtitulación}

En el ámbito del doblaje, Chaume Varela (2012, p. 145) subraya cómo las referencias culturales se convierten en un obstáculo añadido para el traductor, dado que el código lingüístico puede interactuar con los códigos acústico y visual para crear el significado completo de la escena. En ocasiones ocurre lo contrario: son los signos acústicos y visuales los que facilitan la tarea del doblaje de la referencia cultural.

Las estrategias de traducción para el doblaje de estas referencias oscilan entre las que se consideran más extranjerizantes (Venuti, 1995), es decir, las que mantienen los elementos ajenos a la cultura de llegada en la versión doblada, y aquellas que son domesticadoras o, por decirlo de otro modo, se acercan a la cultura de llegada.

Algunas de las estrategias propuestas por Chaume Varela para los textos audiovisuales son comunes a otros textos que no se ven condicionados por la interacción de los macrocódigos: acústico, sintáctico, visual y tecnológico (Casetti y Di Chio, 1991; Chaume Varela, 2004). Por ejemplo, recurrir a la repetición del elemento cultural sin llegar a traducirlo a la cultura término es una de las propuestas de Chaume Varela (2012, pp. 145-146), junto a otras estrategias, como la adaptación ortográfica del elemento cultural sin traducción, la traducción 
literal del elemento cultural de la cultura original, las glosas que permiten que el significado del elemento cultural se haga explícito en la versión traducida, la adaptación o substitución cultural útil para neutralizar los elementos extranjerizantes en la versión traducida, la omisión del elemento cultural o la creación de un nuevo elemento.

La elección de cualquiera de estas estrategias estará condicionada por el texto original (TO), el encargo de traducción, la función del texto en la cultura término (skopos) y las normas de la lengua término.

En relación con la traducción de los elementos culturales en los productos audiovisuales animados, Chaume Varela (2012, p. 146) se remite al trabajo de De Rosa (2010) para afirmar que la animación se sitúa en uno de los dos extremos del continuo extranjerización-domesticación. Es un género particular, debido al tipo de registro lingüístico empleado y al tratamiento que se hace de los elementos culturales. El lenguaje se acerca mucho al discurso oral real, ya que se hallan soluciones traductivas extremadamente domesticadoras, con el propósito de acercar el producto a la audiencia infantil y juvenil.

En el trasvase de los elementos culturales insertos en los productos cinematográficos, Bogucki (2013, p. 71) retoma las posturas de Pedersen (2005), Venuti (1995) y Vinay y Darbelnet (1958) para subrayar que las estrategias propuestas por estos autores podrían emplearse en prácticamente cualquier texto, sea este impreso o visual. Como afirma este autor (Bogucki, 2013, p. 71), la redundancia intersemiótica es la que distingue el contexto de la TAV del contexto impreso. En ocasiones, los macrocódigos que componen el texto audiovisual se superponen y el contenido cultural se repite, lo cual repercute en las decisiones que pueden tomar un doblador o subtitulador.

Ramière (2006, p. 160), a pesar de tener en cuenta las estrategias para el doblaje y la subtitulación compartidas por otras modalidades de traducción (transferencia, traducción literal, explicitación, substitución cultural, neutralización y omisión), defiende que el enfoque debe ser más pragmático y sitúa el contexto como eje de la toma de decisiones del traductor: contexto lingüístico, contexto polisemiótico, pertinencia del elemento cultural en el contexto global de la película, condicionantes técnicos, género cinematográfico, audiencia término, contexto de la distribución (paratextos, por ejemplo), el contexto general cultural y la relación entre la cultura original y la cultura término. Aixelà (1996) puntualiza que la TAV de los elementos culturales depende de la estrategia global escogida, la modalidad de traducción y las normas vinculantes en el entorno sociocultural para el que se traduce.

La subtitulación de los elementos culturales constituye uno de los puntos críticos de la traducción desde el punto de vista de Pedersen (2005), la cual exige la puesta en práctica de varias normas (Toury, 1995), como, por ejemplo, el grado de funcionalismo del texto, el reconocimiento de su skopos, o la creación de una versión del elemento cultural que se acerque más al polo extranjerizador o al domesticador.

Pedersen, además, hace una revisión de las estrategias de traducción que pueden facilitar el trasvase y no traducción — según este autor- del elemento cultural, y cita las siguientes: el uso del equivalente oficial, la retención, la especificación (explicitación y adición), la traducción directa, la generalización, la substitución (substitución cultural), el parafraseo (parafraseo con transferencia del sentido, la paráfrasis situacional) y la omisión.

Si bien es cierto que cada una de estas estrategias puede desarrollarse en profundidad, creemos de mayor interés centrarnos en la opinión de Pedersen respecto a la aplicación de las mismas, ya que las vincula con la práctica profesional del subtitulado. Así pues, este autor afirma que, en la práctica, se tiende a combinar estrategias de forma inconsciente, puesto que gran parte del proceso se lleva a cabo de manera interna. Esto implica que el reconocimiento de las estrategias utilizadas 
para crear la subtitulación de una película, sea de acción real o de animación, puede asociarse con un proceso posterior y reflexivo más que con un elenco de soluciones posibles, de entre las cuales a priori escoge el doblador o subtitulador.

Sin duda, esta reflexión puede aplicarse a la traducción general, aunque Pedersen lo aplica al ejercicio de la subtitulación. De hecho, Pedersen propone siete parámetros que pueden influir en la capacidad de decisión de los subtituladores, que parecen ser de mayor utilidad para la práctica real: el primero de estos parámetros es la transculturalidad (2011, p. 106) que explica cómo los elementos culturales están interconectados entre ellos y cómo ciertos elementos pueden compartirse entre culturas diversas. Se trata de un concepto propuesto por Leppihalme (1994, p. 96) y que puede subdividirse en tres niveles: elemento cultural transcultural (no está vinculado con la cultura del texto origen), elemento cultural monocultural (el referente del elemento cultural no podrá ser identificado por la mayoría de la audiencia de la película subtitulada, debido a que existen diferencias en cuanto al conocimiento enciclopédico compartido en cada cultura) y el elemento cultural microcultural (un elemento que puede escaparse del conocimiento enciclopédico compartido por muchos de los miembros de la cultural original y término).

El segundo parámetro consiste en la extratextualidad (Pedersen, 2011, p. 110) y en si el elemento cultural existe o no fuera del texto original.

La centralidad (2011, p. 111) de la referencia es el tercer parámetro y repercute en la estrategia empleada por el subtitulador. Si el elemento cultural constituye un pilar fundamental de la película, el subtítulo solamente podría retener el elemento cultural o proponer el equivalente funcional de ese concepto cultural. A su vez, pueden hallarse elementos culturales periféricos que no son trascendentales para el desarrollo y la comprensión de la acción fílmica, pero que tal vez sí lo sean desde un nivel microtextual, lo cual impedirá que se efectúe una omisión completa del elemento.
La redundancia intersemiótica (2011, p. 113) tiene que ver con el hecho de que los textos audiovisuales son textos polisemióticos, es decir, son textos que logran transmitir su contenido a través de varios canales y códigos. Dada esta naturaleza del texto audiovisual, los contenidos culturales pueden comunicarse de modo redundante, lo cual permite que el subtitulador proporcione soluciones más simplificadas del elemento cultural presente, por ejemplo, de manera visual y verbal en la película.

Esta redundancia intersemiótica puede relacionarse con el quinto parámetro propuesto por Pedersen, el co-texto (2011, p. 114) que viene a explicar que el subtitulador podrá simplificar e incluso obviar el contenido que se solape en la película. Si el contenido cultural ya se ha explicado en un subtítulo, no tendrá que volver a desarrollarse otra explicación de nuevo en los siguientes subtítulos que hagan referencia al mismo elemento cultural.

Las restricciones propias del medio establecen cómo se produce un cambio semiótico entre la palabra transmitida de manera oral y aquella que toma la forma impresa. Los subtítulos, según Pedersen (2005, p. 13), se han llegado a considerar como una forma híbrida que transmite por escrito ciertos rasgos del lenguaje oral. En consecuencia, la explicitación se convierte en la estrategia empleada con más frecuencia para subtitular los elementos culturales. Pese a esta estrategia, también es cierto que la subtitulación se ve condicionada por las restricciones espacio-temporales (tanto por la extensión del subtítulo como por la duración de la escena en la que se inserta), por lo que la libertad estratégica del subtitulador se ve limitada.

Por último, se deben citar las consideraciones paratextuales (2011, p. 115), que son las que permiten esbozar la globalidad de los objetivos de la traducción que se va a realizar, al tiempo que se establecen cuáles serán las estrategias empleadas genéricamente. Estas consideraciones podrán dilucidarse mediante las directrices disponibles, las agencias de subtitulado, las guías de estilo para las cadenas de televisión o plataformas online, las distribuidoras, etc. 
Tanto las estrategias expuestas hasta aquí como los siete parámetros desarrollados por Pedersen (2005) demuestran que el doblaje y el subtitulado de los elementos culturales entrañan gran complejidad y exigencia. A lo largo de las páginas siguientes se muestra el contenido cultural recopilado y las estrategias que se siguieron para permitir el trasvase interlingüístico.

\section{Método}

Este trabajo pretende analizar el doblaje y el subtitulado de tres películas de animación producidas en España: Planet 51 (Blanco y Abad, 2009), Las aventuras de Tadeo Jones (Gato, 2012) y Atrapa la bandera (Gato, 2015), para conocer qué elementos culturales se han incluido en la versión original de cada una de estas producciones y cómo se han traducido al inglés. Para ello se desarrolla una metodología de trabajo que parte de la clasificación de los elementos culturales propuesta por Katan (1999) y las diversas aportaciones en torno a las estrategias de traducción utilizadas para el doblaje y el subtitulado del contenido cultural.

La elección de este corpus se fundamenta en el interés por conocer qué realidades culturales se insertan en estas películas dirigidas a todos los públicos y cómo estas se doblan y subtitulan para un público de habla inglesa.

Con respecto a la duración de las películas objeto de estudio, Planet 51 dura 82 minutos; Las aventuras de Tadeo Jones, 90 minutos, y Atrapa la bandera, 97 minutos. En total se analizan 269 minutos, que deben visionarse múltiples veces para extraer manualmente los casos, recopilados tanto para hacer el análisis comparativo de la versión original y la doblada como para transcribir los subtítulos de la versión en español y la versión en inglés.

En todas estas producciones participa alguna institución española (principalmente públicas). Por ejemplo, en Planet 51, el Gobierno de España, el MinisteriodeCultura, el InstitutodeCrédito Oficial y el Ministerio de Industria, Turismo y Comercio forman parte del equipo de producción. Esto implica financiación oficial mediante subvenciones o préstamos. En la película protagonizada por Tadeo figuran como productores nacionales Televisió de Catalunya, S. A., el Ministerio de Educación, Cultura y Deporte de España (a través del Instituto de la Cinematografía y de las Artes Audiovisuales, ICAA) y l'Institut Català de les Empreses Culturals (ICEC). En Atrapa la bandera vuelven a colaborar el ICAA y el ICEC, junto al Departament de Cultura y la Generalitat de Catalunya. Estas producciones igualmente han contado con alguna participación de entidades privadas. Así, en la producción de Planet 51 también colaboró el canal de televisión Antena 3, y en Tadeo Jones y Atrapa la bandera, Telecinco Cinema. La implicación de todas estas instituciones nos permite afirmar que la versión original de estas películas es en español, aun cuando pueda existir la participación menor de otras empresas de Estados Unidos o del Reino Unido.

En cuanto a las lenguas que se ofrecen en el soporte disco soporte digital (digital versatile disc, DVD), Planet 51 presenta versiones dobladas al castellano, el inglés, el catalán y el euskera. Las aventuras de Tadeo Jones, en su DVD comercializado en España, solo ofrece el castellano y el catalán como lenguas de visionado. No hay un doblaje al inglés ni al euskera. En el caso de Atrapa la bandera sucede exactamente lo mismo, no hay una pista doblada al inglés ni al euskera. Ninguno de los tres DVD incluye versiones en español de Hispanoamérica, ni en español neutro, ni ninguna otra variedad regional, lo cual restringe el número de espectadores a los que pueden dirigirse estas películas, con la consecuente necesidad de crear versiones específicas en español de Hispanoamérica. Esta decisión comercial tiene repercusiones económicas positivas si implica la contratación de más agentes de doblaje y subtituladores.

Por otra parte, respecto al subtitulado de las películas analizadas, Planet 51 permite la accesibilidad a la cinta gracias a los subtítulos en castellano 
e inglés; Las aventuras de Tadeo Jones solo incluye su subtitulado en español, al igual que Atrapa la bandera. Los subtítulos a los que se accede en español son genéricos. Es decir, la accesibilidad de estas películas se limita, ya que los subtítulos no están dirigidos expresamente a las personas sordas. La audiodescripción tampoco se incluye dentro de las opciones dispuestas para disfrutar de estas películas.

La falta de subtítulos en inglés nos obliga a adquirir los DVD comercializados en el Reino Unido para poder llevar a cabo la investigación que aquí se presenta. Este hecho es bastante inusual, ya que, con frecuencia, los DVD comercializados en España ofrecen la posibilidad de disfrutar de las versiones en inglés (tanto en el doblaje como en el subtitulado de las cintas). Es más, creemos que esta es una de las pocas ocasiones en las que se ha comercializado un producto audiovisual de manera tan restrictiva en este país. La adquisición de los materiales necesarios para realizar esta investigación nos ha centrado inevitablemente en el mercado europeo.

Una vez se solventa esta dificultad metodológica, por la que no todos los productos están disponibles para todos los usuarios de la misma forma, puesto que se adaptan a sus supuestos usuarios, se procede a la recopilación de datos tras el visionado del corpus. Se crea una ficha de trabajo para cada caso extraído. Esta ficha nos permite organizar los datos extraídos en los siguientes epígrafes: código de tiempo (Time Code Record, TCR), tipo de referencia (acústica o visual), contexto de la acción, tipo de referencia cultural, explicación de la misma, versión original doblada, versión doblada, versión original subtitulada intralingüística y versión subtitulada interlingüística.

Cada uno de estos epígrafes nos ofrece una serie de datos que permiten conocer, entre otras cosas, la incidencia del equipo de doblaje en el proceso de trasvase (aquí nos referimos a la medida en la que los agentes de doblaje participan en el trasvase de los elementos culturales hallados en el corpus, teniendo en cuenta los canales por medio de los cuales se transmiten estos elementos), el origen del referente cultural introducido en cada una de las películas y el trasvase entre el español peninsular y el inglés de cada uno de estos referentes, mediante las modalidades de traducción audiovisual aquí tratadas.

La localización de cada escena se facilita gracias a la inclusión del TCR y un breve resumen que contextualiza la acción en la que se incluye el referente cultural. Sabemos, además, que la empresa española Laserfilm Ltd. Cine y Video S. L. se encarga de la creación de los subtítulos de las tres películas. ${ }^{1}$ En los créditos no se menciona a ningún traductor en el caso de Planet 51. Sin embargo, se nombra a Cristina Saur como la responsable de la traducción en Las aventuras de Tadeo Jones y en Atrapa la bandera. Por otra parte, Rafa Russo también trabajó en la traducción de Las aventuras de Tadeo Jones.

La clasificación de cada referente se realiza atendiendo a las categorías descritas por Katan (1999), como se explicó en el apartado teórico anterior. El análisis de cada ficha de trabajo nos proporciona una serie de resultados de carácter cuantitativo y cualitativo, que se desarrollan en este trabajo en el apartado de "Resultados". Antes, proporcionamos más información sobre las películas estudiadas.

\section{Sinopsis de las películas, centrada en la localización del referente cultural}

A modo de resumen, se presenta el contenido temático de cada una de estas películas, con el propósito de orientar al lector en cuanto a qué tipo de referentes culturales se incluyen en el corpus analizado. El contexto de la acción puede ser un indicativo de los referentes de los que se puede hacer uso e incluso puede determinar la aparición de referentes estandarizados (Pedersen, 2005; Toury, 1995)

1 Se ha procedido a la búsqueda de esta información, pero no ha sido posible averiguar si esta empresa realizó también los subtítulos de la versión en inglés. 
a los que se recurre habitualmente en este tipo de películas.

\section{Planet 51}

Esta cinta de animación cuenta la historia de los habitantes de Glipforg, una ciudad que vive cronológicamente en los años cincuenta del siglo $\mathrm{xx}$ estadounidenses. Skiff, amigo del protagonista de la historia, Lem, es una representación del típico joven obsesionado con las películas de alienígenas como Humaniacs III, el próximo estreno al que asistirá. De la ficción cinematográfica, Lem pasa a vivir la experiencia real de conocer a un alienígena terrestre que influirá en su propio desarrollo personal, convirtiéndolo en una persona más aventurera y extrovertida.

Las situaciones que viven Lem, Skiff, Eckle y Neera sirven como ejemplo para el espectador más joven, que es uno de los espectadores tipo al que se dirige esta producción, al que se le enseñan valores de antidiscriminación, convivencia y solidaridad.

Desde el punto de vista de este estudio, el contexto temporal y espacial de esta película proporciona información cultural. La música retoma las producciones propias de los años cincuenta del siglo $\mathrm{xx}$, como los inicios del rock and roll $\mathrm{y}$ algunas baladas. Visualmente, se incluyen referencias a la prensa de la época o a la publicidad tan icónica que se produjo en Estados Unidos durante este periodo. El hecho de que los protagonistas sean adolescentes también influye en qué tipos de referentes culturales se añadirán. Pensamos aquí en la comida y la bebida que consumen, los locales que frecuentan (lo cual influye en la creación de los escenarios para la acción animada), la indumentaria o sus aficiones. Todos estos elementos ya constituyen de por sí posibles elementos culturales y son ajenos a la cultura española. Aun cuando parezcan extraños y alejados, se han introducido mediante la televisión y el cine en la cultura española durante décadas, por lo que los espectadores pueden apreciarlos como foráneos, pero no resultan incomprensibles. Incluso puede que algunos de los espectadores ya hayan asimilado como suyas algunas de estas cuestiones culturales (comida, indumentaria, música, etc.).

\section{Las aventuras de Tadeo Jones}

El propio título de esta película ya nos conduce a su posible temática. Sabemos que será un filme de acción que cuenta con un protagonista inspirado en otro personaje de ficción muy conocido en la industria cinematográfica: Indiana Jones. Esta primera apreciación ya nos permite intuir que la aventura se centrará en el ámbito de la arqueología, como su visionado demuestra.

Tadeo sueña con ser un reputado arqueólogo desde pequeño, hasta que una serie de malentendidos lo llevan a Perú, donde conocerá a Sara Lavrof (toda coincidencia con otra afamada exploradora de ficción es intencionada), hija del arqueólogo al que Jones suplanta accidentalmente. Si añadimos al argumento la presencia de un villano ambicioso que anhela el tesoro de Paititi, ya contamos con los ingredientes que crean un cliché o una parodia de la saga de Lucasfilm Ltd.

Esta película, al igual que la anterior, guarda un espacio a la moraleja o enseñanza final centrada en la importancia del compañerismo y la amistad, más allá de la ambición económica. En esta cinta animada, el contexto espacio-temporal es difuso (y confuso), debido a que el personaje de Tadeo se desplaza entre un entorno españolizado y uno anglosajón. Esto hace que intuir los posibles referentes culturales con anterioridad a la visualización y realizar el análisis de la película sea más complejo.

Por poner un ejemplo de la difuminación de la frontera espacial, Tadeo vive en España, trabaja en la construcción y juega a la lotería, pero visita al profesor en un museo que recuerda al Museo Metropolitano de Arte de Nueva York, y viaja con pasaporte estadounidense desde Chicago a Perú. Este hecho, sin embargo, no preocupará al espectador más joven.

En cuanto a los referentes culturales, es probable que se incorporen referencias relacionadas con 
filmes de acción real similares, en los que la búsqueda del tesoro sea el objetivo del protagonista y del antagonista.

Por otra parte, dado que Tadeo es un adulto, los referentes culturales girarán en torno al viaje, a su profesión, a la vivienda en la que habita o los alimentos que consume.

\section{Atrapa la bandera}

Esta película gira en torno a un descubrimiento histórico que sigue provocando controversia en la actualidad: el día en el que el hombre pisó la luna por primera vez. Los animadores de esta producción trasladan este suceso al ámbito familiar. Sus 3800 planos narran como Mike logra llegar a la luna para curar una herida familiar entre su padre y su abuelo. Además, la aventura cuenta con la presencia de un villano, que pretende ganar la carrera espacial a Estados Unidos.

De nuevo nos encontramos con este proceso narrativo fílmico de difuminación, en el que los personajes creados en España se trasladan a Florida y se infiltran en la Administración Nacional de la Aeronáutica y del Espacio (NASA).

Como en los dos casos anteriores, se trata de una película con mensaje didáctico, en el que la familia lo es todo y en el que se insta a la humanidad a tomar las decisiones correctas por el bien de todos.

Desde el punto de vista de los elementos culturales que pueden intuirse como incluidos en la película, el hecho de que se evoque un acontecimiento histórico como la carrera espacial ya condiciona el tipo de referentes a los que se remitirán los animadores: nombres propios de los astronautas que participaron en la expedición lunar, la inclusión de símbolos y logotipos que pertenecen a instituciones como la NASA, y elementos emblemáticos, como la nave Saturno 5.

Todos estos elementos tienen más relación con la cultura estadounidense que la española, pero, recordemos, esta investigación se centra en el análisis de los elementos culturales hallados en el corpus, su doblaje y subtitulación, sin delimitar ni restringir el origen de estos elementos culturales.

Es cuanto menos llamativo que las producciones españolas incluyan estos elementos ajenos a la propia cultura española, pero vienen determinados por el propio contexto de la película; de ahí que sea muy probable que esta incluya elementos culturales, como la designación de otras películas que narran la exploración de la galaxia o se mencione la prensa de divulgación que pueda abordar esta temática.

\section{Resultados}

La compilación de datos en las fichas de trabajo arroja resultados de carácter cuantitativo y cualitativo. En este sentido, las referencias recopiladas se distribuyeron en 98 fichas y en total se contabilizaron un total de 120 referentes culturales, repartidos del siguiente modo por película: Planet 51, 66 casos; Las aventuras de Tadeo Jones, 32 casos, y Atrapa la bandera, 22 casos. Esta clasificación fue llevada a cabo por ambos autores de esta investigación de manera autónoma e independiente, $\mathrm{y}$ exigió un cuidadoso cotejo de los datos obtenidos. Se comprobó, además, que ambos habían contabilizado el mismo número y tipo de casos y referentes.

\section{Resultados atendiendo al canal}

Con respecto al canal por medio del que se emiten estos casos, 56, es decir, casi la mitad de los casos registrados, se transmiten mediante el canal acústico. Este dato nos indica que tanto para el doblaje como para el subtitulado de estas cintas, el equipo de dobladores/subtituladores desempeña un papel fundamental, porque sin su intervención los diálogos entre los personajes de cada película no serían accesibles a los espectadores ni hispanohablantes (en el caso de la subtitulación) ni a los angloparlantes (para los que el doblaje y el subtitulado se torna la vía de comprensión de las películas animadas estudiadas).

La Tabla 1 refleja cómo se han repartido los elementos culturales hallados en función del canal acústico, del canal visual y el canal compartido acústico y visual. Esta última categoría engloba aquellos casos que no pueden disociarse, es decir, no se aprecia 
Tabla 1 Transmisión de los referentes culturales por película, atendiendo al canal

\begin{tabular}{lccc}
\hline \multicolumn{1}{c}{ Película } & \multicolumn{3}{c}{ Canal } \\
\cline { 2 - 4 } & Visual & Acústico & $\begin{array}{c}\text { Visual y } \\
\text { acústico }\end{array}$ \\
\hline Tadeo Jones & 15 & 17 & 0 \\
Planet 51 & 31 & 30 & 5 \\
Atrapa la bandera & 12 & 9 & 1 \\
\hline
\end{tabular}

con claridad si la referencia se comunica a través del canal acústico o visual en exclusiva.

La transmisión de los contenidos culturales a través del canal acústico revela que el código de significación cinematográfico mayoritario es el código lingüístico. Esto se comprueba tras el análisis de los datos registrados.

Ahora bien, si dirigimos nuestra atención a la detección de los elementos culturales insertados en estas películas, descubrimos, luego de su clasificación atendiendo a las seis categorías descritas por Katan (1999), que la mayoría (74 casos) se incluyen dentro del primer tipo descrito ("medio"). El segundo tipo de referencias culturales, en esta distribución de mayor a menor, se engloba bajo la categoría de "capacidades", seguidas de los referentes que pertenecen al ámbito de los "valores". En la Tabla 2 se muestra cómo se distribuyen los referentes culturales hallados, siguiendo la tipología presentada por Katan (1999).

Tabla 2 Tipos de referentes culturales hallados en el corpus

\begin{tabular}{lcccc}
\hline \multirow{2}{*}{$\begin{array}{c}\text { Referentes } \\
\text { culturales }\end{array}$} & \multicolumn{3}{c}{ Películas } & \multirow{2}{*}{ Total } \\
\cline { 2 - 4 } & $\begin{array}{c}\text { Tadeo } \\
\text { Jones }\end{array}$ & $\begin{array}{c}\text { Planet } \\
\text { Medio }\end{array}$ & $\begin{array}{c}\text { Atrapa la } \\
\text { bandera }\end{array}$ \\
\hline Capacidades & 7 & 44 & 13 & 74 \\
Valores & 4 & 8 & 2 & 17 \\
Comportamiento & 2 & 5 & 4 & 14 \\
Identidad & 1 & 2 & 1 & 8 \\
Creencias & 1 & 1 & 1 & 4 \\
Total & 32 & 66 & 22 & 3 \\
\hline
\end{tabular}

\section{Referentes culturales según el "medio"}

Con el propósito de conocer con detalle cuáles son los referentes culturales utilizados en las películas de animación originales, se procedió a un desglose pormenorizado de la naturaleza de los referentes clasificados bajo la categoría "medio", debido a que es en la que más casos se han recogido. A continuación se muestran los resultados obtenidos por película.

Planet 51. Esta película incluye un número elevado de referencias culturales que se extraen de la creación humana cinematográfica (16 casos), musical (10) y literaria (7 casos). Los otros referentes culturales también se muestran en la Tabla 3.

Tabla 3 Subtipos de elementos culturales (categoría "medio") incluidos en Planet 51

\begin{tabular}{lc}
\hline \multicolumn{1}{c}{ Subtipos de medio } & Casos \\
\hline Cinematográfico & 16 \\
Musical & 10 \\
Literario & 7 \\
Comida & 6 \\
Lugar & 2 \\
Institucional & 1 \\
Moneda & 1 \\
Televisivo & 1 \\
\hline
\end{tabular}

Las aventuras de Tadeo Jones. Los datos extraídos de esta cinta de animación reflejan que las referencias al ámbito cinematográfico vuelven a ser las que superan en número al resto de las halladas: 7 referentes culturales de este tipo (véase Tabla 4).

Tabla 4 Subtipos de elementos culturales (categoría "medio") incluidos en Las aventuras de Tadeo Jones

\begin{tabular}{lc}
\hline \multicolumn{1}{r}{ Subtipos de medio } & Casos \\
\hline Cinematográfico & 7 \\
Comida & 2 \\
Literario & 2 \\
Musical & 1 \\
Publicitario & 4 \\
Televisivo & 1 \\
\hline
\end{tabular}


Atrapa la bandera. De las tres películas analizadas, esta es la cinta de animación que menos casos incluye. Solamente cuenta con 4 referentes del medio cinematográfico. Las demás referencias son escasas (véase Tabla 5).

Tabla 5 Subtipos de elementos culturales (categoría "medio") incluidos en Atrapa la bandera

\begin{tabular}{lc}
\hline \multicolumn{1}{c}{ Subtipos de medio } & Casos \\
\hline Cinematográfico & 4 \\
Histórico & 2 \\
Literario & 2 \\
Lugar & 2 \\
Artístico & 1 \\
Moneda & 1 \\
Publicitario & 1 \\
\hline
\end{tabular}

Estos datos, minuciosamente expuestos, nos demuestran que lejos de estar ausentes, los referentes culturales están muy presentes en las producciomás, esta recopilación de contenido cultural se convierte en una dificultad añadida durante el proceso de traducción, tanto para los agentes del proceso de doblaje como para los subtituladores. El apartado siguiente se centra en qué y cómo se han doblado y subtitulado estas referencias animadas.

\section{Naturaleza de las referencias culturales halladas en la versión en español peninsular: resultados cualitativos}

La compilación y la clasificación de datos de naturaleza cultural extraídos del corpus original permite conocer cualitativamente cuáles son los referentes culturales a los que se recurre para desarrollar el contexto espacial, social y temporal de estas películas. Por ejemplo, en Planet 51, el TO incluye principalmente referencias relacionadas con el contexto aeroespacial, al nombrar las teorías de la conspiración y bases secretas (Base 9, en alusión a la famosa "Área 51" en el desierto de Nevada, en Estados Unidos. Se intuye, además, desde el título de la película y en su temática) o al citar al Centro

Espacial Kennedy o la famosa frase "Houston, tenemos un problema”. Desde el ámbito artístico y creativo, se retoman los mitos literarios sobre alienígenas y monstruos, se cita indirectamente La guerra de los mundos, La guerra de las galaxias hasta en cuatro ocasiones, Encuentros en la tercera fase (traducida así en España), Terminator y Alien. Del mundo televisivo se nombra a los Fraggles (traducida así en España), las televisivas marionetas de Jim Henson, que entretuvieron a una generación de niños y niñas durante los años ochenta y noventa del siglo Xx. Aparece, además, dentro de un subtipo de la categoría medio, una referencia a la prensa impresa, la revista Time, en esta versión animada.

Como suponíamos, se incluyen varias referencias a la comida basura, como chocolatinas, la gelatina, el kétchup y la mostaza, las hamburguesas y bebidas de cola. También hay espacio para referencias españolas como los churros y la canción de Los del Río, Macarena, las cuales ya forman parte de un discurso cultural occidentalizado. Por otra parte, hay un conjunto de referencias aisladas, pero que aportan color local, como los dólares, o menciones al astronauta John Glenn o a Facebook. Termina este listado de referencias la inclusión de ciertas costumbres y el impacto social del movimiento jipi, personificado por Glar Dulam.

En el caso de Las aventuras de Tadeo Jones, las referencias incluidas en la película no están en su totalidad ancladas en la cultura estadounidense. Por ejemplo, Tadeo le cuenta a su jefe que su perro Jeff ha participado en cuatro de los últimos anuncios de la lotería de Navidad (en España y durante varios años, un perro fue el protagonista de varios anuncios de la lotería nacional), a lo que su jefe responde que tiene una noticia para Tadeo y su lotero.

Otras referencias propias de la cultura española son el servicio de transporte y paquetería Seur; la que hasta 2011, tras su fusión con Bristish Airways, fue la aerolínea española de bandera, Iberia; los donuts (donas) y la crema de cacao y 
avellanas Nocilla; la mención a Herodes, para crear la expresión malsonante y coloquial "te jodes como Herodes”, y la famosa frase del actor de doblaje y humorista español José Mota, "Ahora vas y lo cascas". Otras expresiones, en este caso tomadas del lenguaje taurino, también son el origen de escenas con un alto grado de contenido cultural, como sucede cuando Tadeo le pide un capote a Freddy, o cuando este último se lo tira literalmente y le grita “iMaestro!”

En esta película en español, también hay espacio para las referencias culturales anglosajonas. Siguiendo la línea de inclusión de expresiones o frases hechas, se alude a la película Sudden Impact (1983), conocida en España como Impacto súbito, en la que Harry Callahan (Clint Eastwood) dice "Go ahead, make my day". Tadeo cita la traducción canonizada en español de esta película, al decirle a Max Mordon: “Alégrame el día, Max Morro”.

Hay otras referencias culturales de medio que se centran en la creación artística, como, por ejemplo, la réplica de parte de la letra de la canción de Ricky Martin, Maria; la famosa cita de Darth Vader, "Yo soy tu padre"; una referencia a la saga de películas Rambo y, por supuesto, algún guiño a Indiana Jones. Tal vez a modo de homenaje se recuerda a los dibujos animados del pájaro Piolín y el gato Silvestre, gracias al personaje de Freddy, que describe a un puma como "un lindo gatito". Por último, se nombran dos personajes históricos (Einstein y Tutankamón) y dos productos comerciales: la revista National Geographic (como National Petrographic) y la tarjeta de crédito Visa.

Atrapa la bandera solamente cuenta con nueve casos. Se emplean referencias culturales del medio artístico. Se nombra al personaje de Godzilla en dos ocasiones y a la película Alien. Las redes sociales (Facebook y Twitter) como medio de difusión también se añaden a una conversación entre Marty y Amy. Las referencias históricas tienen mayor presencia, posiblemente debido al argumento de la película. Se menciona a Armstrong, su discurso y emplea la palabra mayday (en referencia a la llamada internacional de socorro en navegación y aeronáutica, derivada del francés maider, “ayúdenme”).

\section{La traducción de los elementos culturales: doblaje y subtitulación}

Analizamos, a continuación, los datos referidos al doblaje de los elementos culturales hallados en el corpus, para proceder después al análisis de cómo se efectuó su subtitulación. Se tienen presentes las estrategias de traducción, puesto que nos permiten hacer la comparación entre modalidades de traducción audiovisual.

En Planet 51, las referencias culturales se han doblado manteniendo literalmente el referente en un 84,61\% de los casos. Hay 4 casos que, por lo tanto, han exigido hacer uso de otra estrategia de traducción y que consiste en la sustitución, como se muestra en los ejemplos siguientes. Así, se han recogido dos casos en los que se sustituye la referencia cultural al doblar el código lingüístico al inglés. La "gelatina” y las "gominolas" (ejemplo del caso 1) se sustituyen por "Candy" y "Jelly Beans". También en el ámbito gastronómico se sustituyen el "descafeinado", los "churros" y la "sacarina” (ejemplo del caso 2) por el "frappucino", "puff pastry" y "two sugars". Los "Fraggles" (ejemplo del caso 3) se doblan al inglés como "sea monkeys" y "salir en la tele” (ejemplo del caso 4) se modifica para ofrecer una versión más específica en inglés "Kid’s Choice Awards".

La otra estrategia empleada en el doblaje de Planet 51, más allá de la literalidad y la sustitución, fue la combinación de dos estrategias, es decir, mantener la referencia, aun cuando se omite una parte de la misma. El ejemplo siguiente permitirá comprender mejor cómo se pone en práctica esta estrategia. Chuck conoce a Lem y exclama en español: “iEn el Centro Kennedy van a flipar!”. La versión doblada al inglés omite la palabra "Centro", ya que se espera que el espectador infiera la presuposición cultural y deduzca o identifique que se trata de un instituto tecnológico de investigación: "They're gonna freak back at Kennedy!", nos dice Chuck en inglés. 
La subtitulación de esta película mantiene literalmente el 80,76\% de las referencias culturales recopiladas. Se sustituyen tres referencias culturales, las mismas que las que sustituyeron durante el doblaje. Es decir, la referencia de la gelatina, el café descafeinado y los Fraggles. A su vez, se reproduce, en el texto subtitulado, el caso del Centro Kennedy, que mantiene parte del contenido y omite otra parte en la versión subtitulada. El único caso que experimenta modificaciones entre doblaje y subtitulado se observa en la versión en inglés, en la que la famosa cita se mantiene y expande. La versión doblada al inglés de este fragmento incluso gana en su esfuerzo por crear un lenguaje natural. Chuck dice en inglés (versión doblada): "Houston, we have a little problema". Esta se ha subtitulado como "Houston, we have a little problem".

El doblaje de Las aventuras de Tadeo Jones se realiza mediante el uso de tres estrategias de traducción: la traducción literal, la sustitución y la omisión. De entre los casos estudiados, impera la sustitución como estrategia más usada en el doblaje de esta película (8 casos), lo que supone el 42,10 \% del total. La referencia al anuncio de la lotería de Navidad, tan popular en España, se sustituye por el nombre de uno de los fundadores de la nación estadounidense, Thomas Jefferson, el cual vuelve a reutilizarse en otra secuencia para sustituir a la referencia a Einstein de la versión española. La alusión musical a Ricky Martin y su conocida María también experimenta modificaciones, para convertirse en inglés en Bob Dylan y Rolling Stone. El servicio de transporte y paquetería Seur se adapta a la cultura estadounidense y se modifica por "Fedex". Es lo contrario de lo que se observa para el caso de la inserción de la marca comercial de crédito Visa, mundialmente conocida, pero ausente en la versión traducida. La referencia se sustituye por "savings". A su vez, el personaje de Rambo en inglés se convierte en G.I. Joe y el "Ahora vas y lo cascas" de José Mota se transforma en una referencia cinematográfica a Terminator: "Hasta la vista, baby". La referencia cinematográfica canonizada al español peninsular de la cita "Alégrame el día" de Harry Callahan se sintetiza en inglés: "make me".
La omisión de ciertas referencias culturales en el doblaje de Las aventuras de Tadeo Jones supone un $31,57 \%$ del total de casos recopilados. Las referencias que se pierden en la versión inglesa se corresponden con realidades españolas que podrían haberse sustituido por otras estadounidenses, como ocurre con los casos comentados en el párrafo anterior. Se pierden las referencias a la lotería nacional de Navidad, a la aerolínea Iberia, a los donuts y la Nocilla, al capote y al "lindo gatito", así como la expresión implícita relacionada con el personaje histórico Herodes.

El 26,31\% restante de los casos se corresponden con el uso de la literalidad como estrategia de doblaje, que conserva 4 referencias muy conocidas en la cultura popular occidental por diversos motivos: el faraón Tutankamón, el arqueólogo aventurero Indiana Jones, la famosa cita del villano galáctico Darth Vader: "Yo soy tu padre", y la revista National Geographic. Solamente se ha mantenido una referencia cultural propia de la cultura española; la palabra "maestro", la cual puede ser fácilmente identificable dentro de la cultura término, debido a que, por ejemplo, en Estados Unidos el toreo se conoció gracias a toreros como Manolete.

Por lo que respecta al subtitulado de Tad the Explorer, es decir, de la versión en inglés de Las aventuras de Tadeo Jones, no se pueden aportar datos desde el plano interlingüístico, dado que no se pudo acceder a una versión en inglés de la película que incluyese la versión subtitulada de este filme animado. Se efectuó una revisión de carácter intralingüístico y se comprobó que la versión subtitulada al español mantiene fielmente todas las referencias culturales halladas en el audio original para el español peninsular (19 casos).

Por último, en este análisis contrastivo del doblaje y subtitulado de las referencias culturales incluidas en tres películas de animación de creación española, deben abordarse las estrategias seguidas para el trasvase de la película Atrapa la bandera. De las referencias culturales halladas, se han doblado de manera literal el 77,77 \% de los casos. Tan solo se 
produce un caso de sustitución, en el que el sustantivo con sufijo diminutivo "reporterilla" se dobla como la periodista y novia de Superman, "Lois Lane", creando así una referencia cultural que no existía en el TO. Se incluye, además, una referencia histórica, al utilizar la palabra "mayday" en un contexto en el que estaba ausente.

El subtitulado de esta película mantiene fielmente todos los referentes culturales, así como realiza la sustitución de "reporterilla" por "Lois Lane", como ocurre en el doblaje al inglés de la película.

En términos porcentuales y atendiendo a la totalidad, las versiones dobladas al inglés de las tres películas mantienen, de manera literal, las referencias culturales halladas en un $62,9 \%$. La sustitución permite transferir al inglés el 24,07 \% de las referencias culturales. La omisión impide que se filtren al inglés un $11,11 \%$ de las referencias, mientras que un $1,85 \%$ de las referencias mantienen ciertos aspectos de los referentes culturales, al tiempo que omiten algún elemento de la propia referencia.

En cuanto a la subtitulación de los referentes culturales de dos de las tres películas analizadas destaca la estrategia de traducción literal (80\%). Es decir, los elementos culturales se mantienen entre versión doblada y subtitulada. La sustitución como estrategia para el subtitulado supone el 11,42 \% y la estrategia de mantener ciertos aspectos del elemento cultural y omitir otros representa el 2,85\%.

A modo de curiosidad entre modalidades y versiones, queda un 2,85\% restante, que se corresponde con el caso de la subtitulación del título de la canción del dúo musical Los del Río, Macarena. Esta referencia, que figura en el audio de la versión en español peninsular, no se ha incluido en el subtitulado de la versión en español, la cual podría haberse percibido perfectamente teniendo en cuenta que los discapacitados auditivos pueden sentir el ritmo de la canción e incluso bailar su conocida coreografía. De hecho, la versión subtitulada de esta escena en inglés sí mantiene la referencia a esta canción.

\section{Conclusiones}

Como consecuencia del análisis realizado y de los resultados obtenidos, se concluye este trabajo centrándonos en tres aspectos relacionados con los referentes culturales y su trasvase.

En primer lugar, las 120 referencias halladas demuestran que la animación puede ser rica en conceptos que podrían alejarse de los conocimientos que posee el público infantil y juvenil, lo cual implica diversas lecturas del texto audiovisual y distintos grupos etarios y culturales de receptores. Esto podría servir de base para ulteriores estudios de recepción que contemplasen este condicionante.

Hay que puntualizar, además, que el cine de animación más comercial se dirige habitualmente a todos los públicos y por ello no debe tildarse de sencillo y poco elaborado desde el punto de vista de su contenido visual y lingüístico.

El elevado número de casos recogidosysu clasificación atendiendo al canal de transmisión revela, asimismo, que el doblaje y la subtitulación son fundamentales para hacer accesible el contenido audiovisual de carácter cultural. Sin la participación de los agentes de doblaje y los subtituladores, las referencias culturales no constituirían los únicos contenidos perdidos en el trasvase entre lenguas y culturas.

En cuanto a la naturaleza de las referencias halladas, y este es el segundo aspecto concluyente, se observa que estas están ligadas al argumento de cada película, a su contexto espacial y temporal. Se detecta, por otra parte, una miscelánea con respecto al origen de estas referencias; es decir, no todas son de origen español, sino que muchas pertenecen al ámbito anglosajón, en concreto, a Estados Unidos. Sorprende que estas producciones españolas incorporen este tipo de referentes ajenos y opinamos que detrás se hallan razones económicas y de mercadotecnia. En España (y posiblemente también en otros mercados hispanos), la película se venderá mejor si parece estadounidense, aun cuando los fondos con los que se produce son españoles, y en Estados Unidos su aceptación estará garantizada, 
porque los referentes culturales son propios y prácticamente universales. En otras palabras, la elección e inclusión de determinados referentes culturales aparece intencionadamente desde la fase de producción, y está mediatizada por el potencial de distribución en el contexto cultural término de la película, una vez traducida a la lengua de la cultura término.

Los aspectos y las decisiones traductológicas del proceso traslativo se situarían así en un momento anterior en el proceso de realización de la película. Cabría, en este sentido, analizar y constatar la posible existencia de este planteamiento en otras producciones, tanto de animación como de otros géneros, lo que podría implicar nuevos enfoques sobre el análisis de los procesos de traducción, tanto en traducción audiovisual como en otros ámbitos, como la traducción literaria.

Por último, tras el análisis cuantitativo, puede concluirse que la estrategia de traducción empleada con mayor frecuencia para el doblaje y el subtitulado de los referentes culturales recopilados fue la traducción literal. Este dato nos demuestra que entre doblaje y subtitulado, al menos para este corpus, no existen diferencias en cuanto a la difusión del contenido cultural, y que el subtitulado mantiene, a pesar de sus restricciones espaciales, la carga cultural expresada en la versión doblada.

La sustitución o adaptación cultural es la segunda estrategia utilizada con más frecuencia $(24,07 \%)$, seguida de un índice muy inferior de omisiones $(11,11 \%)$. Desde un punto de vista centrado en el consumidor de estas películas, resulta alentador observar cómo el contenido cultural está al alcance de todos los espectadores, lo cual habrá supuesto un esfuerzo mayor para los subtituladores de este cine animado. Esto constituye, sin duda, un mérito adicional que merece ser destacado.

\section{Referencias}

Aixelà, J. F. (1996). Culture-specific items in translation. En R. Álvarez y C. Vidal (Eds.), Translation, power, subversion (pp. 52-78). Multilingual Matters.
Baker, M. (1992). In other words. A coursebook on translation. Routledge.

Bassnett, S. (1991). Translation studies. (2. ${ }^{\text {a }}$ ed.). Routledge.

Blanco, J., y Abad, J. (Directores) (2009). Planet 51 [Película]. TriStar Pictures et al.

Bogucki, Ł. (2013). Areas and methods of audiovisual translation research. Peter Lang. https://doi. org/10.3726/978-3-653-03263-5

Casetti, F., y Di Chio, F. (1991). Cómo analizar un film. Paidós. Chaume Varela, F. (2004). Cine y traducción. Cátedra.

Chaume Varela, F. (2012). Audiovisual translation. Dubbing. St. Jerome.

Cómitre Narváez, I. (2015). Culture-bound aspects in subtitling of animated films. Tales of the night of $M$. Ocelot. https://riuma.uma.es/xmlui/handle/10630/9798

Cómitre Narváez, I., y Valverde Zambrana, J. M. (2014). How to translate culture-specific ítems: A case study of tourist promotion campaign by Turespaña. The Journal of Specialised Translation, (21), 71-112.

De Rosa, G. L. (2010). Dubbing Cartoonia. Mediazione interculturale e funzione didattica nel processo di traduzione dei cartoni animate. Loffredo Editore.

Gato, E. (Director) (2012). Las aventuras de Tadeo Jones [Película]. El Toro Pictures et al.

Gato, E. (Director) (2015). Atrapa la bandera [Película]. Paramount Animation et al.

Hermans, T. (1999). Translation in systems. Descriptive and systemic approaches explained. (Translation Theories Explained, Series 7). St. Jerome.

Katan, D. (1999). Translating cultures. An introduction for translators, interpreters and mediators. St. Jerome.

Leppihalme, R. (1994). Culture bumps. On the translation of allusions. University of Helsinki, English Department Studies 2.

Leppihalme, R. (1997). Culture bumps. Multilingual Matters.

Mayoral Asensio, R. (1994). La explicitación de la información en la traducción intercultural. En A. Hurtado Albir (Ed.), Estudis sobre la traducció (pp. 73-96). Universitat Jaume I.

Nedergaard-Larsen, B. (1993). Culture-bound problems in subtitling. Perspectives, 1(2), 207-242. https://doi. org/10.1080/0907676X.1993.9961214

Newmark, P. (1988). A textbook of translation. Prentice Hall.

Pedersen, J. (2005). How is culture rendered in subtitles? En Mutra 2005-Challenges of Multidimensional 
Translation: Conference Proceedings. http://www. euroconferences.info/proceedings/2005_Proceedings/2005_Pedersen_Jan.pdf

Pedersen, J. (2011). Subtitling norms for television. John Benjamins Publishing Company.

Ramière, N. (2004). Comment le sous-titrage et le doublage peuvent modifier la perception d'un film. Analyse contrastive des versions sous-titrée et doublée en français du film d'Elia Kazan, A streetcar named desire (1951). Meta, 49(1), 102-114. https://doi. org/10.7202/009026ar

Ramière, N. (2006). Reaching a foreign audience: Cultural transfers in audiovisual translation. Journal of Specialised Translation, (6), 152-166.
Snell-Hornby, M. (1988). Translation studies. An integrated approach. John Benjamins. https://doi. org/10.1075/z.38

Toury, G. (1995). Descriptive translation studies - and beyond. John Benjamins. https://doi.org/10.1075/btl.4

Vandeweghe, W. (2005). Duoteksten. Inleiding tot vertaling en versaalstudie. Academia Press.

Venuti, L. (1995). The translator's invisibility. A history of translation. $\quad$ Routledge. https://doi. org/10.4324/9780203360064

Vinay, J.-P., y Darbelnet, J. (1958). Comparative stylistics of French and English. (J. C. Sager y M.-J. Hamel, Trad. y ed.). John Benjamins.

Cómo citar este artículo: Fuentes-Luque, A., y López González, R. (2020). Cine de animación made in Spain: doblaje y subtitulación de elementos culturales. Íkala, Revista de Lenguaje y Cultura, 25(2), 495511. https://doi.org/10.17533/udea.ikala.v25n02a08 